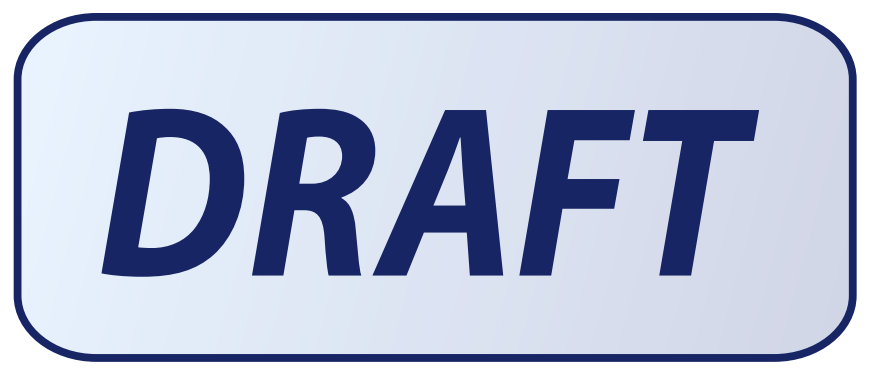

\title{
Postural stability control for Robot-Human cooperation for sit-to-stand assistance.
}

\author{
V. Pasqui*, L. Saintbauzel and P. Bidaud \\ Université Pierre et Marie Curie-Paris6, FRE 2507, ISIR \\ 18 Route du Panorama 92 265 Fontenay-aux-Roses \\ *E-mail: pasqui@robot.jussieu.fr \\ http://lrp6.robot.jussieu.fr/ \\ J. Graefenstein \\ Leibniz University of Hanover \\ E-mail: j.graefenstein@web.de
}

\begin{abstract}
This article presents a fuzzy controller, for a robotic device, to ensure stability of the user during the assisted sit-to-stand transfer. The first problem to be addressed is the postural analysis of the chair rising. Experiments, with healthy subjects, were performed with this aim in view. Analysis of external forces shows that sit-to-stand transfer can be subdivided into several phases. The observation of the Center of Pressure and of the horizontal component of the handle force yields rules to observe the stability of the patient and consequently adjust the robotic interface motion to the human volontary movement. These rules are used in the fuzzy control implementation. The controller is validated on experiments with healthy subjects and diseaded patients.
\end{abstract}

Keywords: Assistive device; robotic interface; human centered robotic; postural stability; sit-to-stand; fuzzy control.

\section{Introduction}

The aim of the work presented in this paper is to realise a robotic interface for equilibrium assistance during Sit-To-Stand (STS) transfer. Here, it is supposed that interactive robotic devices, as human-centered robotics, is more comfortable and more efficient that traditionnal technical devices [1].

Robotics technologies have been investigated in the last few years to prevent falls by a postural control of patients and to promote safe mobility [2], [3], [4], $[5],[6],[7],[8],[9]$. But these robotic devices have no postural correction to restore equilibrium.

Based on an analysis of the most common walking troubles associated with aging or cerebellar syndrome we have designed and developed a robotic device (in Figure 1) [10] to help the patient to sit-down, stand-up and walk [11]. The assistive device handles guide the patient to rise from a chair or to sit down, 


\section{DRAFT}

following trajectories which are based on parameters reflecting personal strategies [12].

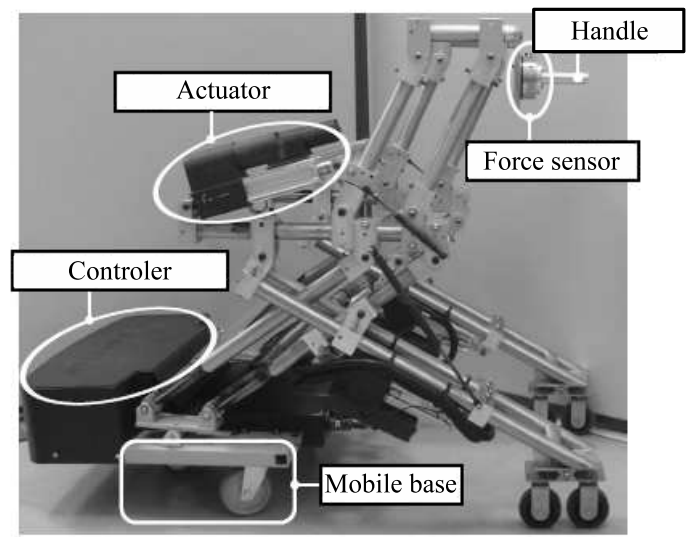

Fig. 1. Robotic Interface prototype

We propose here to detail how an adapted control can give interactive ability to this robotic interface.

\section{Method}

By interactivity, we intend the capacity to interpret the postural movements detected by the sensors to trigger the movement or to maintain the postural equilibrium.

To observe the postural state, experimental dynamical analysis of the stand-up have been done in our laboratory (Figure 2) . Results show different phases of chair rising, that are matching with physiological litterature [13]. Each phase depends on interaction forces between human and handle : $\vec{F}_{h}=\left(F_{h x}, F_{h y}\right)$; or human and ground : $\vec{F}_{g}=\left(F_{g x}, F_{g y}\right)$ and their time variations. Reaction force between human and ground is computed at Center of Pressure (CoP) which position may be used as a stability criteria [14].

The observation of the CoP position and direction of the force $\vec{F}_{h}$ yields simple rules to identify unstability cases or desired movement to trigger (i.e. beginning of the STS). Fuzzy controller is used in intention detection to control neural prostheses [15] or orthosis using FES [16].

Fuzzy controller seems to be a good way for interactivity, then we have extended the role of the fuzzy controller from the detection of voluntary movement to the detection of the unstability.

The fuzzy control has to fulfill two tasks, thats defined two output:

- output 1: recognition of the current phase,

- output 2 : determination of proper reaction to ensure stability of subject. 


\section{DRAFT}
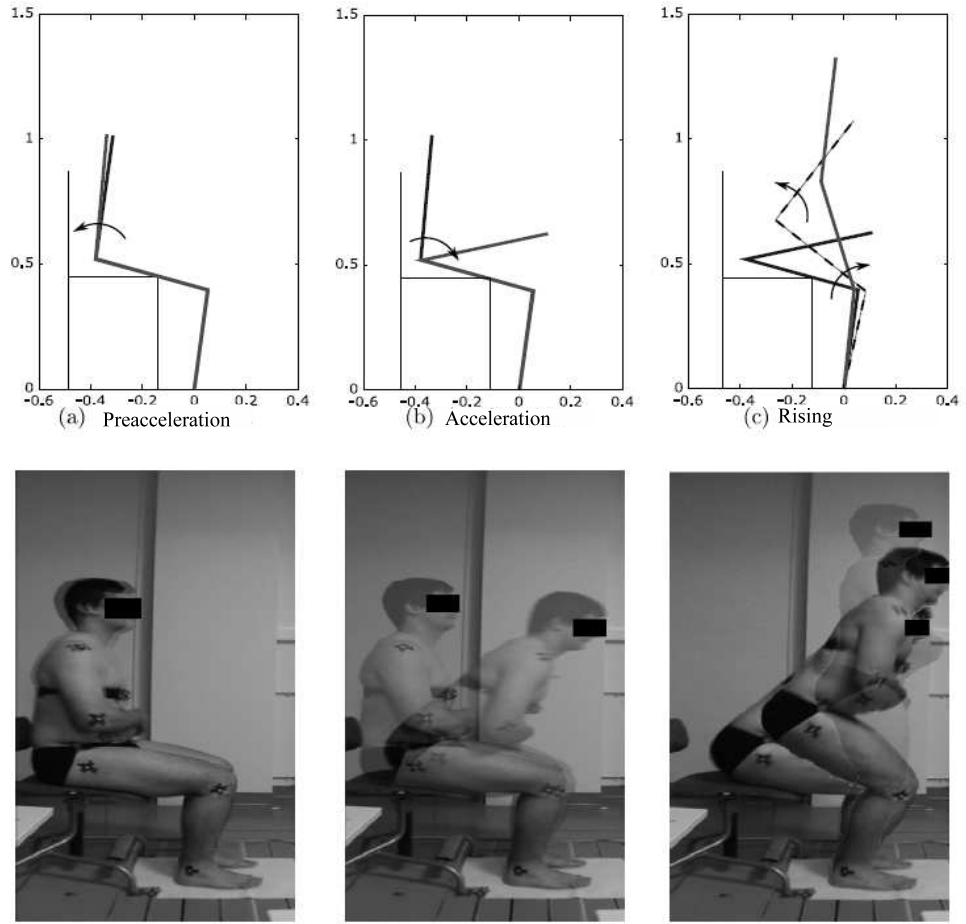

Fig. 2. Different sit-to-stand phases analysis

The following fuzzy sets were defined for the output 1 (in Figure 3 ) : seated, returned, preaccelaration (preac), acceleration (acc), start rising (startr), rise. The detection of the phases of the STS is obtained analysing the value of the $\vec{F}_{h}, \vec{F}_{g}$ and the time variation of $\vec{F}_{h}$.

"Returned" identifies the case when subject aborts stand-up and returns to the seated position.

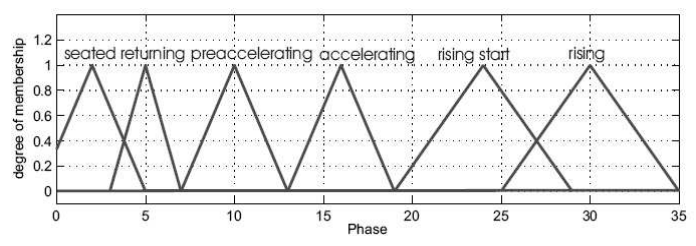

Fig. 3. Membership functions for output 1 


\section{DRAFT}

The membership functions for the output2, that determines the movement, if proper phase is detected, are shown in Figure 4.The following fuzzy sets were defined:

instable (inst) : object underlies high unbalance. Quick reaction is required. stabilize (stab): object indicates desire of stabilization.

no move (nm): no movement is necessary in the horizontal direction. adjust (adj) : object desires another position of the handles.

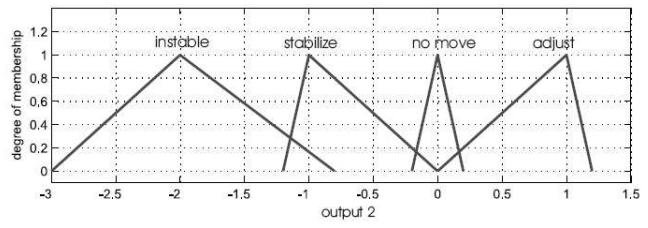

Fig. 4. Membership functions for output 2

If we denote $\mathrm{H}$ for high, $\mathrm{Z}$ for zero, $\mathrm{L}$ for low, $\mathrm{EL}$ for extremly low and $\mathrm{EH}$ for extremly high, we have for exemple :

IF $F_{g y}=E L A N D F_{h x}=L A N D \frac{d F_{h y}}{d t}=H$ THEN the human is RISING.

IF $F_{h x}=H A N D C o P=L$ THEN the human posture is stable.

The rulebase for the fuzzy control is presented in the table figure 5 .

\begin{tabular}{|c|c|c|c|c|c|c|c|c|c|c|c|}
\hline$F_{g x}$ & $F_{g y}$ & $\frac{d F_{g x}}{d t}$ & $\frac{d F_{g y}}{d t}$ & $F_{h x}$ & $F_{h y}$ & $\frac{d F_{h x}}{d t}$ & $\frac{d F_{h y}}{d t}$ & $\mathrm{CoP}$ & $\frac{d \text { CoP }}{d t}$ & out 1 & out 2 \\
\hline$Z$ & $Z$ & - & - & $Z$ & $Z$ & - & - & - & - & seated & $\mathrm{nm}$ \\
\hline$H$ & - & $H$ & - & $H$ & 0 & $H$ & - & - & - & preacc & $\mathrm{nm}$ \\
\hline$L$ & - & $E H$ & $L$ & - & - & - & $H$ & - & - & accel & - \\
\hline- & $E L$ & - & - & $L$ & - & - & $H$ & - & - & rise & - \\
\hline$H$ & $E L$ & $Z$ & $Z$ & $L$ & - & $Z$ & $Z$ & - & - & rise & - \\
\hline$L$ & $E L$ & $H$ & $E L$ & $L$ & $H$ & - & - & - & - & startr & - \\
\hline$E H$ & $E H$ & $H$ & $H$ & $H$ & $H$ & - & - & - & - & return & - \\
\hline- & - & - & - & $H$ & - & - & - & $L$ & - & - & stab \\
\hline- & - & - & - & $L$ & - & - & - & $L$ & - & - & adj \\
\hline- & - & - & - & $H$ & - & - & - & $L$ & - & - & stab \\
\hline- & - & - & - & $H$ & - & - & - & $H$ & - & - & adj \\
\hline- & - & - & - & $Z$ & - & - & - & $Z$ & - & - & nm \\
\hline- & - & - & - & $Z$ & - & - & - & - & $E L$ & - & inst \\
\hline- & - & - & - & $Z$ & - & - & - & - & $E H$ & - & inst \\
\hline
\end{tabular}

Fig. 5. Rulebase for the fuzzy control 


\section{DRAFT}

Detection of unstable posture is illustrated in figure 6 , where both patient and robot are modelised by a 3 links model each. The difference between these two models is in the interaction with ground. We assume that robotic interface cannot loose contact with ground while patient could if he is unstable.
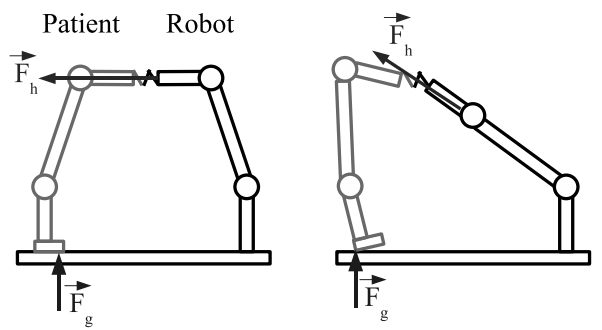

Fig. 6. Interaction between patient and robotic interface

If a subject, under perturbations, is verge on to loose his balance, he quickly shifts the load within the foot support area in the opposite direction of fall direction. If the impending fall directed forwards, the $\mathrm{CoP}$ will rapidly move in the same direction. An according reaction could be observed for a fall backwards.

\section{Application}

The complete structure of the controller is shown in figure 7 .

The preprocessing block receives forces measurement, applies a filter and calculates the position of $\mathrm{CoP}$ and its time derivatives. These outputs are processed by the fuzzy logic block to identify patient posture state. Then, the corresponding control mode is selected between those:

- Normal : tracking trajectory,

- Impedance : Impedance control according to the efforts of interactions measured,

- Stabilization : modification of the tracking trajectory to stabilize the patient,

- Return : the interface returns to the initial position,

The movement is triggered by the preacceleration phase.

For cases identified as a patient aborting movement, the robot returns to the initial position. If postural unstability is detected the device motion in vertical direction is stopped and a new desired position is computed that guarantee patient stability.

\section{Results}

The presented prototype is currently in Bellan Hospital for a rehabilitation protocol validation. Many diseaded patients, with cerebellar syndrome, have tested the device. This kind of pathology imposes a ballast walking-aid to filter 


\section{DRAFT}

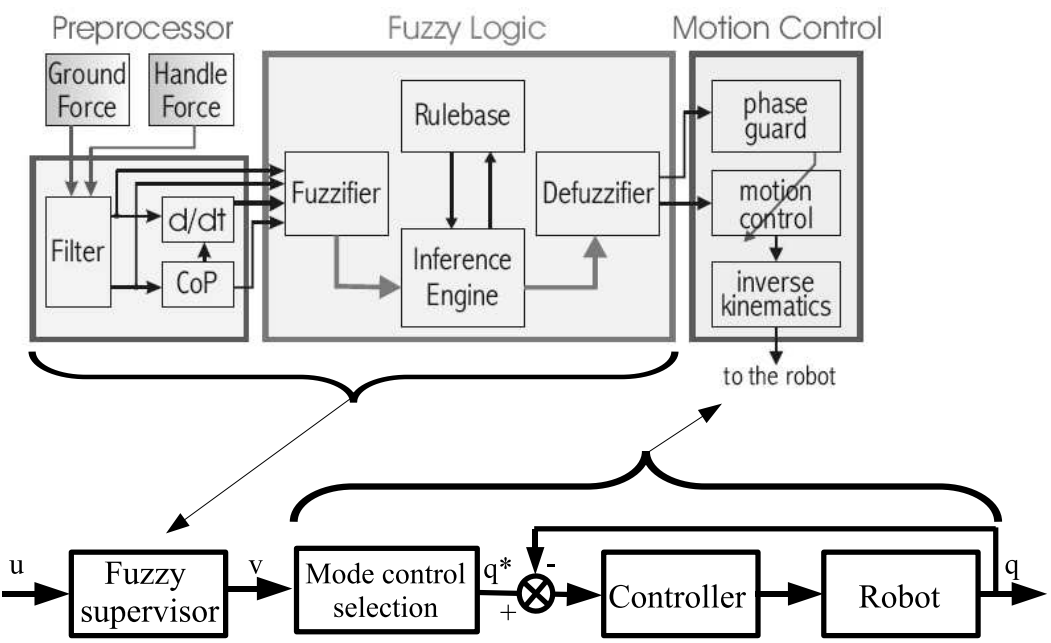

Fig. 7. Control structure
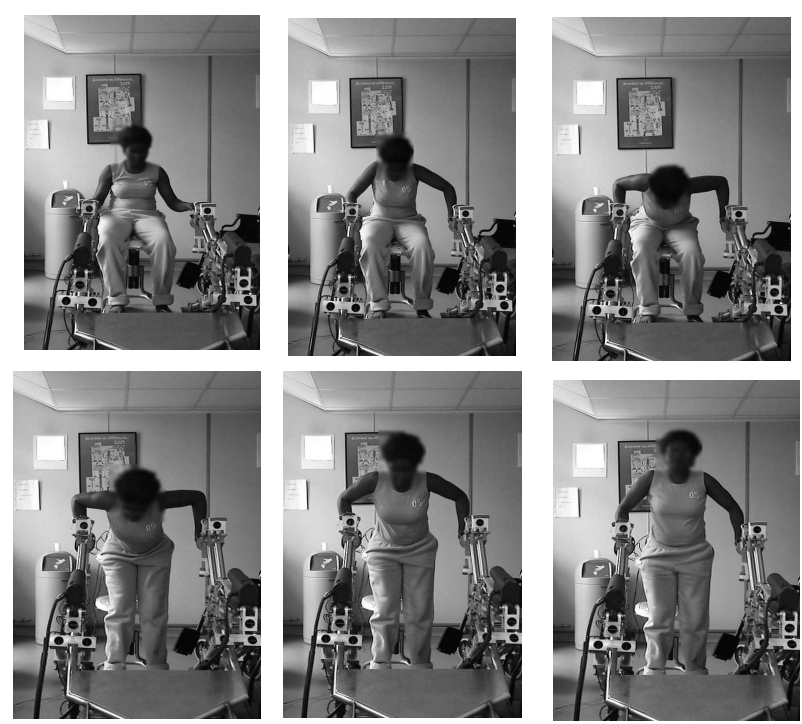

Fig. 8. Prototype in Bellan Hospital

shaking. The robotic interface is more comfortable, less tiresome and easy to drive. In addition to assist in position change and walking, this device can detect onset of fall. Then the robotic interface will response by changing handle 


\section{DRAFT}

position, producing a force to balance the patient, as it is shown in Figure 9.

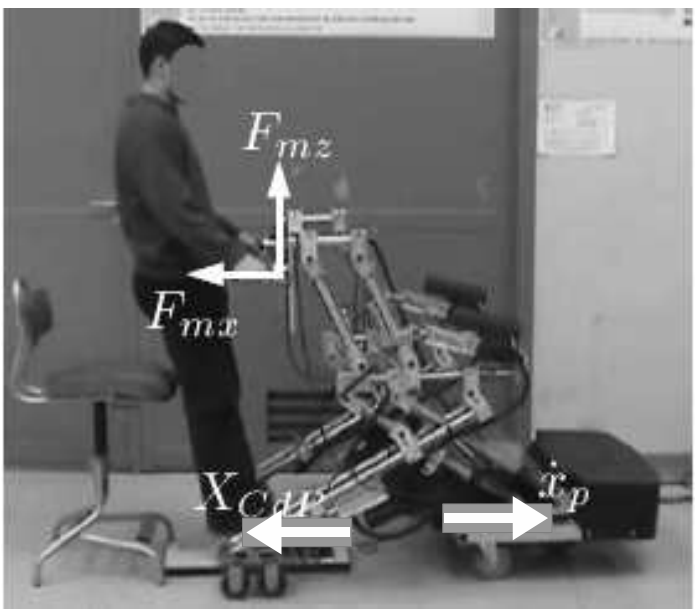

Fig. 9. Unstable posture corrected by the robotic interface

\section{Conclusion}

Fuzzy logic is very usefull for detection of movement intention and unstable postures. Provided with a fuzzy supervisor, the robotic interface becomes highly interactive.

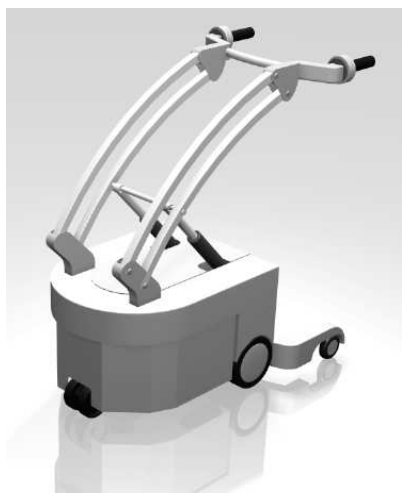

Fig. 10. A commercial product 
Now, objectives are to develop rehabilitation protocols with collaboration with the medical team of Bellan Hospital. A second objective is to produce a commercial product with ROBOSOFT society. Such a product would be designed as in Figure 10.

\section{References}

1. R. reiner, L. Lunenburger and G. Colombo, human-centered robotics applied to gait training and assement., in Journal of rehabilitation research and development, (5) 2006 .

2. S. C. Lee, K. Oh K. and J. Lee, A system for gait rehabilitation : Mobile manipulator approach., in Proc. IEEE Int. Conference on Robotics and Automation, (ICRA'02), (Washington, USA, 2002).

3. K. Nagai, I. Nakanishi and H. Hanafusa, Assistance of self-transfer of patients using a power-assisting device., in Proc. IEEE Int. Conference on Robotics and Automation, (Taipei, Taiwan, 2003).

4. Korean eldercare robots are coming. (2006), http://robotgossip. blogspot.com/2006/07/korean-eldercare-robots-are-com\%ing. html.

5. M. Spenko, H.-Y. Yu and S. Dubowsky, A robotic personal aid for the mobility and health monitoring for the elderly., in IEEE Trans on Neural Systems and Rehabilitation Engr, 2007.

6. N. Rea, G. Lacey, C. Lambe and R. Dahyot, Multimodal periodicity analysis for illicit content detection in videos., in The 3rd European Conference on Visual Media Production (CVMP 2006), 2006.

7. B. Graf, M. Hans and D. S. Rolf, Care-o-bot ii development of a next generation robotic home assistant., in Autonomous Robots, 2004.

8. J. Pineau, M. Montemerlo, M. Pollack, N. Roy and S. Thrun, Towards robotic assistants in nursing homes : Challenges and results., in Robotics and Autonomous Systems, (3-4)

9. H. Park, H. Hong, H. Kwon and M. Chung, A nursing robot system for the elderly and the disabled., in International Journal of Human-friendly Welfare Robotic Systems, (4)

10. P. Médéric, V. Pasqui, F. Plumet and P. Bidaud, Sit to stand transfer assisting by an intelligent walking-aid, in Proc. 7th International Conference on Climbing and Walking Robots, (CLAWAR'04), (Madrid, Espagne, 2004).

11. P. Médéric, V. Pasqui, F. Plumet and P. Bidaud, Elderly people sit to stand transfer experimental analysis, in Proc. 8th International Conference on Climbing and Walking Robots, (CLAWAR'05), (London, UK, 2005).

12. V. Pasqui and P. Bidaud, Bio-mimetic trajectory generation for guided arm movement during assisted sit-to-stand transfer, in Proc. 9th International Conference on Climbing and Walking Robots, (CLAWAR'06), (Bruxel, Belgium, 2006).

13. R. Aissaoui and J. Dansereau, Biomechanical analysis and modelling of sit to stand task: a literature review, in Proceedings of IEEE International Conference on Systems, Man, and Cybernetics, 1999.

14. P. Sardain and G. Bessonet, Forces acting on a biped robot. center of 
pressure-zero moment point, in IEEE Transactions on Systems, Man and Cybernetics, (5)2004.

15. P. C. Sweeney, G. M. Lyons and P. H. Veltink, Finite state control of functional electrical stimulation for the rehabilitation of gait, in Medical and Biological Engineering and Computing, (2)2006.

16. S. Hussein and G. M.H., Intention detection using a neuro-fuzzy emg classifier, in IEEE Engineering in medecine and biology, 2002. 